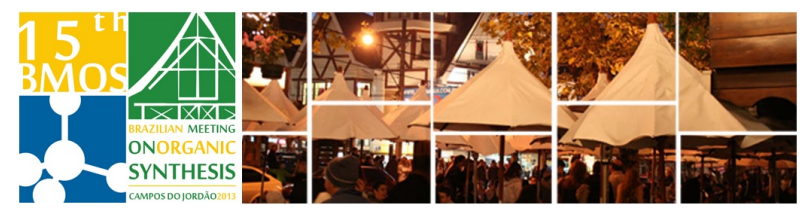

\title{
Synthesis and Evaluation against Mycobacterium tuberculosis of Phenazine-triazole-linked from ortho-Naphthoquinones
}

\section{Guilherme A. M. Jardim, ${ }^{a}$ Gleiston G. Dias, ${ }^{a}$ Emilay B. T. Diogo, ${ }^{a}$ Diego A. C. Silva, ${ }^{a}$ Bernardo L. Rodrigues, ${ }^{a}$ Jéssica Cantos, ${ }^{b}$ Pedro E. A. Silva ${ }^{b}$ and Eufrânio N. da Silva Júnior $^{\mathrm{a}^{*}}$}

aInstituto de Ciências Exatas, Departamento de Química, UFMG, Belo Horizonte, MG, Brazil; ${ }^{b}$ Laboratório de Micobacterias, Faculdade de Medicina, FURG, Rio Grande, RS, Brazil..

*eufranio@ufmg.br

Keywords: Lapachone, Quinone, Mycobacterium tuberculosis, Click chemistry.

\section{INTRODUCTION}

Phenazine compounds have been reported as new active substances againt Mycobaterium Tuberculosis strains. ${ }^{1}$ In the last years, click chemistry reaction was used to incorporate a 1,2,3-triazole ring in bioactive heterocyclic compounds, as for instance, bioactive phenazines. $^{2}$ This strategy, known as molecular hybridization is important in medicinal chemistry for potentiate the pharmacological activities and reduce the sides effects. ${ }^{3}$

In this context, we described herein the synthesis and evaluation against Mycobacterium tuberculosis of new phenazine-triazole-linked.

\section{RESULTS AND DISCUSSION}

The first class of compounds were prepared from $\beta$ lapachone. $\beta$-lap was reacted with $N$-bromosuccinimide (NBS) in $\mathrm{CCl}_{4}$ with benzoyl peroxide as initiator to produce 3,4-dibromo- $\beta$-lapachone. This compound was used to prepare 3 -bromo-4-azide- $\beta$-lapachone $(\mathbf{1})$, then $\mathbf{1}$ was submitted to azide-alkyne Huisgen's cycloaddition catalyzed by $\mathrm{Cu}(\mathrm{I})$, producing the respective 1,2,3triazolic compounds 2-6. By simple reaction with orthophenylenediamine the respective phenazines 7-11 from quinones 2-6 were obtained in high yields (Scheme 1).

The structures of the compounds novel 7-11 were determined by ${ }^{1} \mathrm{H}$ and ${ }^{13} \mathrm{C}$ NMR, IR and mass spectrometry. For compound $\mathbf{9}$ suitable crystals were obtained and the structures of this compound was reconfirmed by X-ray crystallography data studies. ORTEP3 projection is showed in the Figure 1.
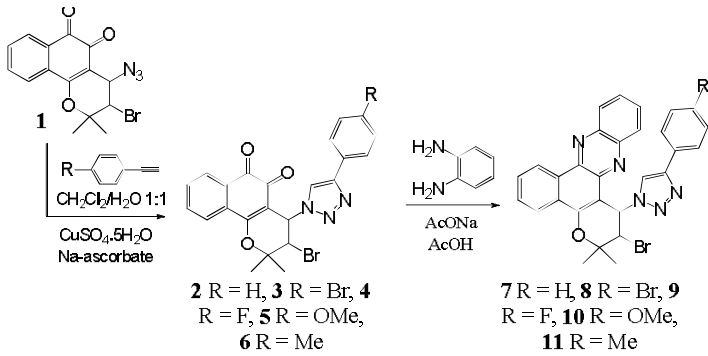

Scheme 1. Synthesis of the phenazine-triazole-linked 7-11

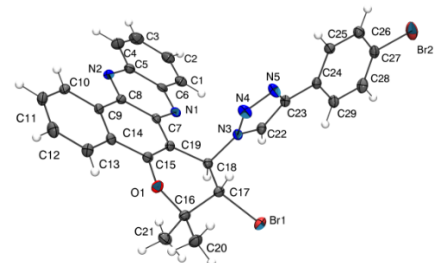

Figure 1. An ORTEP-3 projection of molecule 9 showing the atomnumbering and displacement ellipsoids at the $20 \%$ probability level.

Following the same methodology phenazines 19-24 were obtained from the 3 -azido-nor- $\beta$-lapachone (12) that was previously prepared as described in the literature (Scheme 2). All the substances were well characterized and synthesized in high yields. The phenazines herein described for the first time were evaluated against the agent that cause tuberculosis (TB) and were found good activities. For merit of simplification the data were not showed.

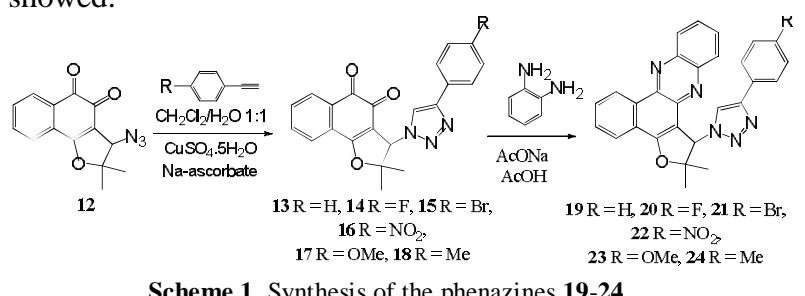

\section{CONCLUSION}

New prototypes used against TB have been successfully obtained and well characterized and these compounds can be considered as possible novel antimicrobial derivatives.

ACKNOWLEDGEMENTS
CNPQ, CAPES, FAPEMIG, UFMG and FURG.
REFERENCES
1. Salas, C. O et. al.. Curr. Med. Chem. 18, 2011, $144-$
161.
2. da Silva Júnior, E. N. et. al. Euro. Jour. Med. Chem.
46, 2011, 4521 - 4529.
3. Gmeiner, P. J. et. al. Med. Chem. 2009, 52, 6860.

